\title{
Targeted Delivery of a Novel Conjugate Nef-M1 Peptide and Single-Walled Carbon Nanotubes promotes Growth Inhibition and Mortality for Breast and Colon Tumorspheres
}

\author{
Harvey L. Bumpers ${ }^{1 凶}$, Janagama D. Goud ${ }^{2}$, Upender Manne ${ }^{3}$, Venkat R. Katkoori ${ }^{*}$ \\ 1. Department of Surgery, Michigan State University, East Lansing, MI, USA \\ 2. Independent Research Scientist, Atlanta, GA, USA \\ 3. Department of Pathology, University of Alabama at Birmingham, Birmingham, AL, USA \\ *Senior Author \\ $\square$ Corresponding author: Harvey L. Bumpers, MD, FACS, Professor of Surgery, Department of Surgery, College of Human Medicine, Michigan State \\ University, 660 South Hagadorn Rd, Suite 600, Lansing, MI 48823. Office (517) 267-2460; Fax (517) 267-2488; harvey.bumpers@hc.msu.edu \\ (c) Ivyspring International Publisher. This is an open access article distributed under the terms of the Creative Commons Attribution (CC BY-NC) license \\ (https://creativecommons.org/licenses/by-nc/4.0/). See http://ivyspring.com/terms for full terms and conditions.
}

Received: 2018.10.26; Accepted: 2019.01.22; Published: 2019.03.25

\begin{abstract}
The Nef-Ml peptide (Nef-Ml) has been shown to be an inhibitor of growth and metastasis of breast cancer (BC) and colorectal cancer (CRC) cells. Since a nano-construct for Nef-Ml could enhance its efficacy, we developed three-dimensional (3-D) cultures of tumorspheres of $B C$ and $C R C$ cells and treated them with functionalized, single-walled carbon nanotubes (SWNTs) conjugated with Nef-MI (SWNTs-Nef-MI) to evaluate inhibition of cell growth. We hypothesized that cancer cells cultured as tumorspheres would be more sensitive to SWNTs-Nef-Ml than to Nef-Ml alone. 3-D cultures of human BC cells (MDA-MB-231 and MDA-MB-468) and CRC cells (SW480) were developed with 1\% Sea Prep Hydrogel in supplemented RPMI-1640 medium. SWNTs-Nef-MI was prepared by use of thionine, and its structure was confirmed by ultraviolet (UV) spectral analysis and gel electrophoresis. The tumorspheres were treated with Nef-MI or SWNTs-Nef-MI to compare their relative effects. Internalization of SWNTs-Nef-MI was evaluated by transmission electron microscopy. The viability/apoptosis status of the tumorspheres was established by use of ethidium bromide and acridine orange staining using fluorescent microscopy. Gel electrophoresis and UV spectral analysis confirmed formation of the SWNTs-Nef-MI conjugate. After 3 weeks, 3-D cultures of BC and CRC cells developed as tumorspheres, which, in the presence of Nef-MI, showed reduced growth and increased apoptosis. This effect was greater in the presence of SWNTs-Nef-MI, consistent with enhanced delivery of Nef-Ml to the cells via SWNTs. This sets the stage for more detailed evaluation to quantify apoptosis and elaboration of the mechanism of increased apoptotic activity. Delivery of an apoptotic peptide, Nef-MI, into 3-D cultures of BC and CRC cells was achieved via a nanoparticle construct with SWNTs. Furthermore, compared to Nef-MI, SWNTs-Nef-Ml suggests more extensive apoptosis.
\end{abstract}

Key words: single-walled carbon nanotubes, Nef-M1 peptide, 3-D cultured tumorspheres, cancer therapy, drug delivery, breast and colon cancers

\section{Introduction}

In therapeutic oncology, nanocargo-based drug targeting is a promising modality. Functionalized single-walled carbon nanotubes (SWNTs) act as nanoneedles, penetrate into cells, and attach to subcellular structures without apparent cytotoxicity to the cell. SWNTs can be functionalized to react with 
the carboxylic/amide groups of proteins, peptides, DNA, RNA, oligonucleotides, sugar moieties, or polyoxide derivatives [1-5]. SWNTs, which are efficient vehicles for delivery of molecules, have been tested for drug and vaccine delivery, lymphatictargeted chemotherapy, thermal therapy, photodynamic therapy, gene therapy, and suppression of tumor growth through gene interference [6, 7]. SWNTs can carry nano-cargos of diagnostic and therapeutic molecules to the sites of interest for cancer diagnosis and targeted therapy [8-14]. Carbon nanotubes, in particular single-walled nanotubes, interact with the dye, thionine [15]. Relative to covalent functionalization, functionalization of SWNTs with thionine is simple, convenient, rapid, and cost-effective for targeted cancer therapy. SWNTs conjugated with small interfering RNAs (siRNAs) are internalized by tumor cells, wherein they release siRNA to silence target genes [7]. In the present study, we functionalized SWNTs with thionine and subsequently attached an apoptotic peptide, Nef-M1, to the amidated SWNTs.

The Nef (negative regulatory factor) gene of the human immunodeficiency virus (HIV) encodes a 27-34 kD myristoylated protein, which is expressed after establishment of the provirus in host cells. Nef-M1, which is cytotoxic to a variety of human cancer cells, functions as an activator of apoptosis and an inhibitor of cancer cell growth and metastasis [16, 17]. Nef-M1 competes with stromal-cell-derived factor 1a (SDF-1 $\alpha)$, the natural ligand for chemokine (C-X-C motif) receptor-4 (CXCR4), and induces apoptosis [18]. Nef-M1 is a potential inhibitor of both tumor growth and metastasis [17]. Based on these findings, we hypothesized that treatment of BC and CRC tumorspheres with SWNTs conjugated with Nef-M1 (SWNTs-Nef-M1) will be an effective strategy for inhibition of their growth and increased mortality through enhanced cytotoxicity. Therefore, in the present study, we developed tumorspheres of BC and CRC cells, treated them with SWNTs-Nef-M1, and evaluated the efficacy of this agent in inhibition of cell growth.

\section{Materials and Methods}

\section{Cell cultures}

BC (MDA-MB-231, and MDA-MB-468) and CRC (SW480) cell lines (ATCC, Global Resource Center, Manassas, VA) were determined to be free of pathogens and mycoplasma. These cells were maintained in RPMI-1640 medium (ATCC) supplemented with 10\% fetal bovine serum (FBS, Thermo Scientific HyClone, Logan, UT), 25 mM HEPES, penicillin/streptomycin
(Pen/Strep, Mediatech, Manassas, VA), L-glutamine $(0.3 \mathrm{~g} / \mathrm{L})$, and sodium bicarbonate $(2 \mathrm{~g} / \mathrm{L})$, and incubated at $37^{\circ} \mathrm{C}$ with $5 \% \mathrm{CO}_{2}$. All cell lines were initially grown as monolayers. These cells were used for development of three-dimensional (3-D) cultures. Briefly, cells were mixed with $1 \%$ Sea Prep Hydrogel (Lonza, Rockland, USA) in Nonclon petri dishes (5-cm diameter). The preparations were allowed to solidify for 30 minutes at $4^{\circ} \mathrm{C}$. Subsequently, RPMI- 1640 culture medium supplemented as described above was added to the gels with embedded cells. The cells were allowed to grow at $37^{\circ} \mathrm{C}$ with $5 \% \quad \mathrm{CO}_{2}$ for formation of tumorspheres.

\section{Light and fluorescent microscopic characterization of tumorspheres}

The tumorspheres were visualized with light and fluorescent microscopes. For testing of viability, the cells were harvested, washed, and diluted with $1 \mathrm{x}$ phosphate-buffered saline (1xPBS) to $1-5 \times 10^{6}$ cells $/ \mathrm{mL}$. Equal amounts $(25 \mu \mathrm{L})$ of tumorsphere suspension and ethidium bromide and acridine orange were incubated for 10 minutes at room temperature (RT) and visualized with blue light by use of fluorescent microscopy (495-nm primary filter and a 515-nm secondary filter). Live cells fluoresced green (with acridine orange), and dead/apoptotic cells fluoresced orange (with ethidium bromide).

\section{Preparation of SWNTs-Nef-MI}

Thionine can be used as functional molecule for the non-covalent functionalization of SWNTs, as it shows a strong interaction with SWNTs. Attachment of thionine onto SWNTs would improve the solubility and lower the thermal stability of original SWNTs. Thionine may functionalize the surface of SWNTs with rich $\mathrm{NH} 2$ groups and therefore open up more opportunities for the surface chemistry of SWNTs. Targeting ligands including antibodies and peptides can be conjugated to functionalized SWNTs to recognize specific cell receptors. Nef-M1 is a peptide that binds specifically to the CXCR4 receptor over-expressed on a wide range of human cancer cells. To prepare functionalized SWNT-Nef-M1 conjugate, first SWNTs were (Sigma-Aldrich, St. Louis, MI) sonicated and treated with $37 \%$ hydrochloric acid. For adsorption and binding of thionine to SWNTs, an aqueous suspension of SWNTs was sonicated with thionine (Sigma-Aldrich) solution $(10 \mathrm{mg} / \mathrm{mL}$ ) at RT for 12 hour. The preparations were then filtered with a $1.2-\mu$ diameter pore membrane. The purple color of the thionine solution disappeared, indicating that thionine reacted with the SWNTs. The SWNTs/thionine solutions were stored at $4^{\circ} \mathrm{C}$ until 
time of use. The SWNTs amidated with thionine were washed, treated with $20 \%$ glutaraldehyde, and reacted with Nef-M1 $(1 \mathrm{mg} / \mathrm{mL})$ with shaking for 1 hour. The peptide attached onto SWNTs via a condensation reaction with thionine. Gel electrophoresis $(10 \%$ polyacrylamide) followed by Coomassie dye staining was performed to assess the binding of Nef-M1 with SWNTs.

\section{Treatment of tumorspheres with SWNTs-Nef-MI}

On the $7^{\text {th }}$ day and $12^{\text {th }}$ day of $3-\mathrm{D}$ culture, tumorspheres of BC and CRC cells in 96-well plates were treated with Nef-M1 or SWNTs-Nef-M1 conjugate ( $2 \mu \mathrm{g} /$ well) to determine the effects of these agents on their growth. The viability status of the tumorspheres was assessed by use of ethidium bromide or acridine stain and fluorescent microscopy.

\section{Characterization of internalized SWNT-Nef-MI}

To confirm internalization of SWNT-Nef-M1 in the cells, a JOEL 1200EX transmission electron microscope (TEM) (JEOL Ltd., Japan) was used. Cells were grown on cover-slips, washed with $1 \times$ PBS, fixed in primary $(2.5 \%$ glutaldehyde) and secondary $(1 \%$ osmium tetroxide) fixatives, block stained,

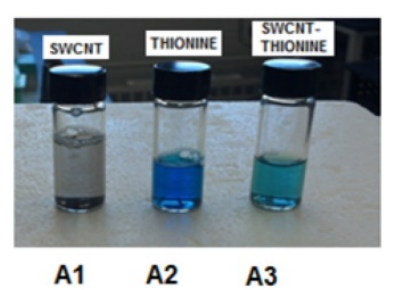

C

SWNT - Thionine conjugate (A3)

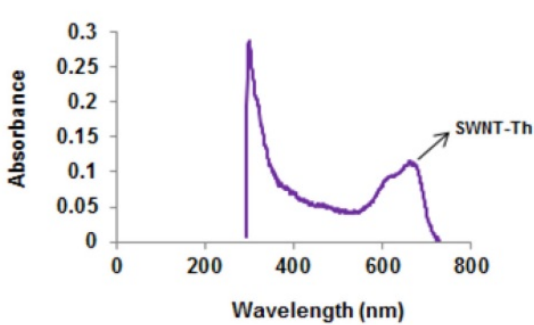

Figure 1. Nef-M1 functionalized with SWNTs. (A). Disappearance of the purple color of the solution showed that carbon nanotubes adsorbed thionine molecules. (B). Aqueous solutions of thionine had adsorption peaks around $600-700 \mathrm{~nm}$ and $250 \mathrm{~nm}$. (C). Two peaks were evident in the SWNT-thionine conjugate (A3) indicating linkage of thionine molecules onto SWNTs. (D). Gel electrophoresis shows the formation of SWNTs-Nef-MI complex which is illustrated in Lane 1 and the individual component in Lane 2. dehydrated and passed through a transitional solvent (propylene oxide), resin filtered, sectioned and visualized under TEM at $80 \mathrm{KV}$.

\section{Results}

\section{3-D cultures developed as tumorspheres}

$B C$ and CRC cells were initially grown as 2-D cultures. These cells were then harvested and cultured with $1 \%$ Sea Prep hydrogel and carbon cobalt particles in supplemented RPMI-1640 medium using nanomagnetic levitation as previously reported by us [19]. After 3 weeks the 3-D cultures developed as tumor spheres that were suspended in the Sea Prep hydrogel. Similar to in vivo conditions, these 3-D cultures developed cell-cell interactions and the cells within the spheres are held together by an extracellular matrix. The cells of 3-D culture were found to have changed in morphology and formed spheres that linked to form microtissue noted in suspension.

\section{Nef-MI functionalized with SWNTs}

It was important to confirm that Nef-M1 developed a stable link to SWNT, showing enhanced function of the conjugate over the individual components. This binding of Nef-M1 with SWNTs was confirmed by UV spectral analysis and gel electrophoresis followed by staining with Coomassie blue. The solubility of the SWNTs when linked with thionine increased its ability to form the conjugate. In addition, the purple color of the solution disappeared (Figure 1A), implying that the carbon nanotubes adsorbed thionine molecules. UV-visible absorption measurements were employed to explore the interactions between SWNT and thionine. Figures 1B \& C shows the UV-visible absorption spectra of thionine and thionine-modified SWNTs suspended solutions. The spectrum of pure thionine had two chaacteristic adsorption peaks: one in visible region around $600-700 \mathrm{~nm}$ and the other around $270 \mathrm{~nm}$ in UV region. The two peaks were also evident for thionine-modified SWNTs, indicating the presence of 
thionine molecules on SWNTs. Gel characterization showed that the Nef-M1 peptide was attached to SWNTs functionalized by thionine (Figure 1D, lane 1).

\section{Effect of SWNTs-NefMI conjugates on growth of tumorspheres}

In 96-well plates, tumorspheres of BC and CRC cells were treated with SWNTs, Nef-M1 or SWNTsNef-M1 $(2 \mu \mathrm{g} /$ well $)$ on day 7 and day 12 of 3-D cultures growth. SWNTs-Nef-M1 was internalized by cancer cells that express CXCR4 receptors, a target of Nef-M1, but also by cells not expressing CXCR4 receptors (Figure 2), indicating that SWNTs carrying the Nef-M1 entered into both types of cells. With Nef-M1 treatment, the viability of 3-D tumorspheres, as determined by ethidium bromide or acridine staining and fluorescent microscopy showed a reduction of growth and increased disintegration (apoptosis). Treatment with SWNTs-Nef-M1 indicated by visual inspection that enhanced cellular mortality occurred as compared to treatment with either SWNTs or Nef-M1 and this was consistent with increased delivery of Nef-M1 into the cells via the conjugate.
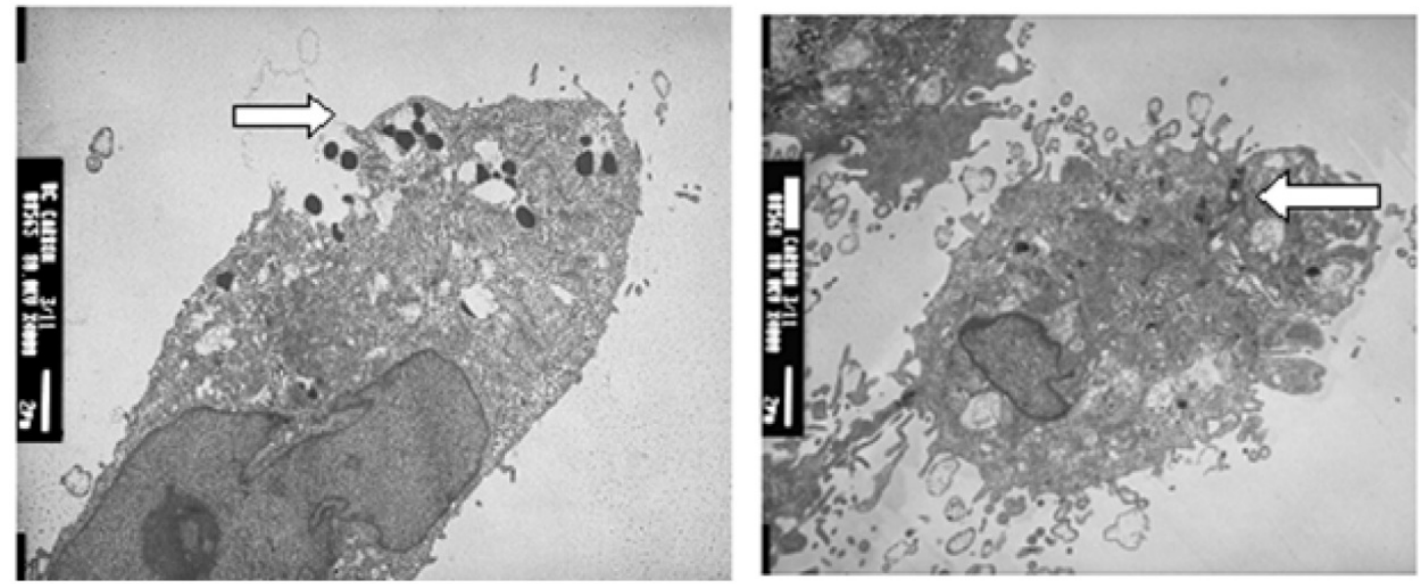

Figure 2. SWNT-NefMI was internalized by cells. Transmission electron micrographic (TEM) images of MDA-MB-231 (positive for CXCR4 receptors) and MDA-MB-468 (negative for CXCR4 receptors) cells with internalized SWNT-Nef-M1, indicating entry of the complex into both cell types.
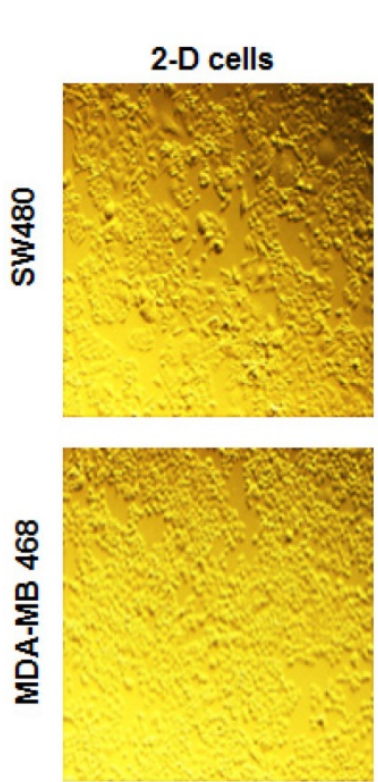

A
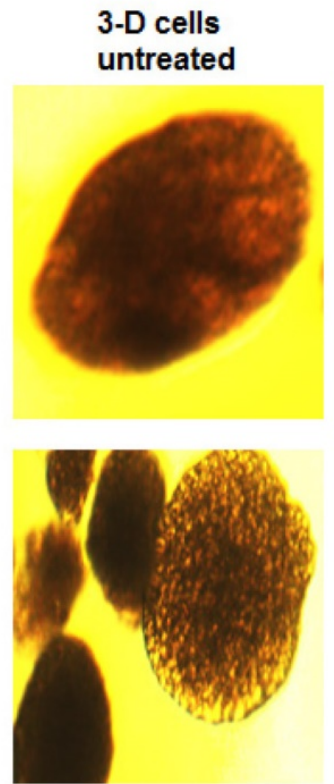

B

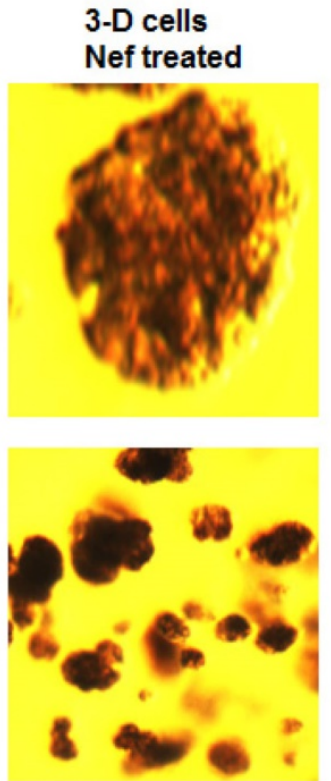

C
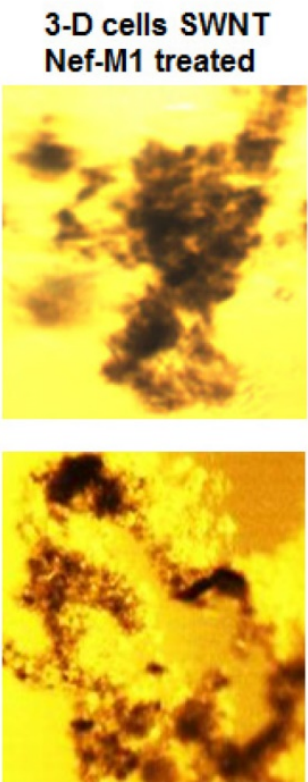

D

Figure 3. SWNT-NefMl suppresses growth of tumorspheres. Human BC (MDA-MB-468) and CRC (SW480) cells were grown in monolayers (A) and in 3-D cultures untreated (B). The tumorspheres were untreated, treated with Nef-M1 (C), or treated with SWNTs-Nef-M1 (D). Nef-M1 caused reduction of growth and disintegration of BC and CRC tumorspheres. SWNTs -Nef-M1 caused enhanced effects, indicating delivery of Nef-M1 into the cells via SWNTs. The 2D images at 10x magnification to illustrate confluent growth on the flask. The other images at 40x magnification shows the 3D tumorspheres formation. 


\section{Discussion}

Relative to monolayer cell cultures, 3-D model systems more accurately mimic BC and CRC biology $[19,20]$. Thus, the 3-D models represent an intermediate step between primary cell culture and animal models. We hypothesized that 3-D cultures would provide a model for assessing delivery of a potentially therapeutic apoptotic peptide, Nef-M1, attached to SWNTs. In the present study, we developed 3-D cultures of BC and CRC cells and determined the effect of Nef-M1 and SWNTs-Nef-M1 on the growth of tumorspheres. The cells of 3-D cultures, which are held together by an extracellular matrix [20,21], had morphology similar to those of tumors in animals, offering an environment closer to that of tumors growing in animals and allowing delivery of nano-peptide conjugates.

Carbon-based nanoconfigurations, such as carbon nanotubes and nanofibers, have received considerable attention for cancer treatment [22-24]. This is due to their distinctive features, including high mechanical strength and surface area, sites for chemical or physical conjugation (enhancing functionalization), light-weight properties, and a capacity to penetrate mammalian cells and deliver small peptides, proteins, and RNA or DNA molecules. However, issues related to their biocompatibility, renal clearance, and toxicity has limited their use in targeting cancer. For instance, as a result of systemic administration, most inhibitors of angiogenesis often fail to reach the targeted tumor vessels due to poor biodistribution, short half-lives, and side effects [25]. An advance has come from using nanotechnology to target tumor-associated vasculature [26] and thereby affect tumor growth and metastasis. These advantages are made possible by features, including the size, shape, and functional aspects of nanoparticles that allow them to target metastases, and act as a nano needle to enter the cells, and be retained. In endothelial cells, differential activation of avb3 integrins and downstream phosphoinositide 3- kinase (PI3K) signaling by nanotubes of various shapes implicate the role of nanotube configuration in mediating drug fate and underlies the importance of choosing an appropriate vector to obtain optimal delivery of the active peptide (optimal therapeutic index) [27-30]. For these reasons, nanotherapeutics is emerging as a new approach for cancer treatment.

Non-covalent binding to enhance functionalization appears to be an alternative to covalent functionalization. Non-covalent functionalization largely retains the intrinsic optical properties of SWNTs, which are useful in various biological imaging and sensing applications. SWNTs with adsorbed thionine molecules have elevated solubility and decreased thermal stability [31]. These conjugating products have been useful in linking various molecules onto nanotube surfaces for delivering in to cells. In the present study, gel characterization showed that Nef-M1 could be effectively attached onto the SWNTs. The attachment was achieved via a condensation reaction between thionine and Nef-M1 molecules. These conjugates were used to inhibit growth in BC and CRC cultures.

Peptides are attractive targeting molecules due to their small size, low immunogenicity, and low cost [32]. Peptide-based targeting ligands may be identified via several methods. Most commonly, they are obtained from the binding regions of a protein of interest. Cilengitide, a cyclic peptide with integrin binding affinity, underwent phase III clinical trials for the treatment of glioblastoma [33, 34], Adnectin, a 40-amino acid, thermostable and protease-stable oligopeptide attached to an inhibitor of human VEGF receptor 2 (Angiocept) has entered phase II clinical trials for treatment of recurrent glioblastoma multiforme [35]. Although peptides have disadvantages, such as a low target affinity and susceptibility to proteolytic cleavage, these issues may be ameliorated by stabilizing the peptides multivalently or utilizing D-amino acids [36]. Recently, peptides have been attached to functionalized SWNTs for targeted cancer therapy [10].

In tumors, CXCR4 is a target receptor, because it is highly expressed in various cancers, including BCs and CRC [37-44], and expression of this receptor has been shown to correlates with tumor progression. Using therapeutic agents to target CXCR4 provides an attractive strategy for the treatment of cancer. Nef-M1 competes with the CXCR4 natural ligand, SDF-1a, and induces apoptosis [18]. It is cytotoxic to various cancer cells and is a potential inhibitor of cancer cell growth and metastasis [16, 17]. By virtue of the Nef-M1 affinity for the CXCR4 receptor there is potentially a therapeutic cellular selectivity. This conjugate, SWNT-Nef-M1, further enhances toxicity since the CXCR4 receptor is upregulated in BC and CRC and the SWNT exposes those cells to higher amounts of the Nef-M1. Like most cytotoxic chemotherapies some level of non-selectivity occurs and benign cells may be affected to some degree. However, the presence of the receptor and increase volume of the apoptotic peptide (Nef-M1) delivered to the cancer cells produces a higher cytotoxicity. This is confirmed in culture as there is extensively more cell death in malignant culture than in benign one.

With Nef-M1 treatment, tumorspheres of BC and CRC cells showed a reduction of cell growth and increased disintegration. SWNTs-Nef-M1 produced a greater effect, indicating better delivery of Nef-M1 
into the cells by this nanoparticle conjugate. More studies are necessary to better understand if this conjugate enhances toxicity by increasing the amount of the functionalized peptide that is delivered or if the conjugate upregulates the effectiveness of the receptor binding to increased apoptosis.

\section{Acknowledgements}

We thank Dr. Donald L. Hill, Comprehensive Cancer Center, University of Alabama at Birmingham, AL, for his critical review of this manuscript. This work was supported in part by NIH/NCI Workforce Diversity Grant R21-CA171251 to HLB and by U54 CA118948 to HLB and UM.

\section{Competing Interests}

The authors have declared that no competing interest exists.

\section{References}

1. Huang W, Taylor S, Fu K, Lin Y, Zhang D, Hanks TW, et al. Attaching proteins to carbon nanotubes via diimide activated amidation. Nano Lett. 2002. 2: 311

2. Pantarotto D, Briand JP, Prato M, Bianco A. Translocation of bioactive peptides across cell membranes by carbon nanotubes. Chem Commun (Camb). 2004. 1:16.

3. Williams KA, Veenhuizen PT, de la Torre BG, Eritja R, Dekker C. Nanotechnology: carbon nanotubes with DNA recognition. Nature. 2002. 420 (6917): 761.

4. Nguyen CV, Delzeit L, Cassell AM, Li J, Han J, and Meyyappan M. Preparation of nucleic acid functionalized carbon nanotube arrays. Nano Lett. 2002. 2 (10): 1079.

5. Pompeo F, and Resasco D. Water solubilization of single-walled carbon nanotubes by functionalization with glucosarine. Nano Lett. 2002. 2 (4): 369.

6. Huang N, Wang H, Zhao J, Lui H, Korbelik M, Zeng H. Single-wall carbon nanotubes assisted photo thermal cancer therapy: animal study with a murine model of squamous cell carcinoma. Lasers Surg Med. 2010. 42 (9): 638.

7. Zhang Z, Yang X, Zhang Y, Zeng B, Wang S, Zhu T, et al. Delivery of telomerase reverse transcriptase small interfering RNA in complex with positively charged single-walled carbon nanotubes suppresses tumor growth. Clin Cancer Res. 2006. 12 (16): 4933.

8. Shim M, Kam NWS, Chen RJ, Li Y, and Dai H. Functionalization of carbon nanotubes for biocompatibility and biomolecular recognition. Nano Lett. 2002. 2 (4): 285.

9. Sun $\mathrm{YP}, \mathrm{Fu} \mathrm{K}$, Lin $\mathrm{Y}$, Huang $\mathrm{W}$. Functionalized carbon nanotubes: properties and applications. Acc. Chem. Res. 2002. 35: 1096.

10. Chen J, Chen S, Zhao X, Kuznetsova LV, Wong SS, Ojima I. Functionalized single-walled carbon nanotubes as rationally designed vehicles for tumor-targeted drug delivery. J. Am. Chem. Soc. 2008. 130: 16778.

11. Dhar S, Liu Z, Thomale J, Dai H, Lippard SJ. Targeted single-wall carbon nanotube-mediated $\mathrm{Pt}(\mathrm{IV})$ prodrug delivery using folate as a homing device. J. Am. Chem. Soc 2008. 130: 11467.

12. Bhirde AA, Patel V, Gavard J, Zhang G, Sousa AA, Masedunskas A, et al. Targeted killing of cancer cells in vivo and in vitro with EGF-directed carbon nanotube-based drug delivery. ASC NANO. 2009. 3: 307.

13. Heister E, Neves V, Tilmaciu C, Lipert K, Sanz Beltra V, Coley HM, et al. Triple functionalisation of single-walled carbon nanotubes with doxorubicin, a monoclonal antibody, and a fluorescent marker for targeted cancer therapy. Carbon. 2009. 47: 2152.

14. Honga $\mathrm{H}, \mathrm{Gao} \mathrm{T}$, Cai W. Molecular imaging with single-walled carbon nanotubes. Nano Today. 2009. 4: 252.

15. Li Q, Zhang J, Yan H, He M, Liu Z. Thionine-mediated chemistry of carbon nanotubes. Carbon. 2004. 42 (2): 287.

16. Harrington $\mathrm{W}$, Bond V, Huang MB, Powell M, Lillard J, Manne U, et al. HIV Nef-M1 effects on colorectal cancer growth in tumor induced spleen and hepatic metastasis. Mol Cell Pharmacol. 2009. 1(2): 85.
17. Bumpers HL, Huang MB, Powell M, Grizzle WE, Lillard JW Jr, Okoli J, et al. Effects of HIV-1 Nef, a cytotoxic viral protein, on the growth of primary colorectal cancer. Cancer Biol Ther. 2005. 4: 65

18. Huang MB, Jin LL, James CO, Khan M, Powell MD, Bond VC. Characterization of Nef-CXCR4 interactions important for apoptosis induction. J Virol. 2004. 78: 11084.

19. Bumpers HL, Janagama DG, Manne U, Basson MD, Katkoori V. Nanomagnetic levitation 3-D cultures of breast and colorectal cancers. J Surg Res. 2015. 194: 319.

20. Martin KJ, Patrick DR, Bissell MJ, Fournier MV. Prognostic breast cancer signature identified from 3-D culture model accurately predicts clinical outcome across independent datasets. PLoS ONE. 2008. 3 (8): e2994.

21. Nyga A, Cheema U, Loizidou M. 3D tumour models: novel in vitro approaches to cancer studies. J. Cell Commun. Signal. 2011. 5: 239.

22. Ji SR, Liu C, Zhang B, Yang F, Xu J, Long J, et al. Carbon nanotubes in cancer diagnosis and therapy. Biochimica Biophysica Acta. 2010. 1806: 29.

23. Liu Z, Chen K, Davis C, Sherlock $\mathrm{S}$, Cao Q, Chen X, et al. Drug delivery with carbon nanotubes for in vivo cancer treatment. Cancer Res. 2008. 68 (16): 6652.

24. Elhissi AMA, Ahmed W, Israr Hassan UI, Dhanak VR, and D'Emanuele A. Carbon nanotubes in cancer therapy and drug delivery. J Drug Deliv. 2012. 2012: 1

25. Harold F and Vorak D. Vascular permeability factor/vascular endothelial growth factor: A critical cytokine in tumor angiogenesis and a potential target for diagnosis and therapy. J Clin Oncol. 2002. 20: 4368

26. Peer D, Karp JM, Hong S, Farokhzad OC, Margalit R, Langer R. Nanocarriers as an emerging platform for cancer therapy. Nat Nanotechnol. 2007. 2: 751.

27. Yang F, Jin C, Yang D, Jiang Y, Li J, Di Y, et al. Magnetic functionalized carbon nanotubes as drug vehicles for cancer lymph node metastasis treatment. Eur J Cancer. 2011. 47 (12): 1873.

28. Dhar S, Liu Z, Thomale J, Dai H, Lippard SJ. Targeted single-wall carbon nanotube-mediated $\mathrm{Pt}$ (IV) prodrug delivery using folate as a homing device. J Am Chem Soc. 2008. 130 (34): 11467.

29 Hampel S, Kunze D, Haase D, Krämer K, Rauschenbach M, Ritschel M, et al. Carbon nanotubes filled with a chemotherapeutic agent: a nanocarrier mediates inhibition of tumor cell growth. Nanomedicine. 2008. 3 (2), 175

30. Liu YT, Zhao W, Huang ZY, Ye XY. Noncovalent surface modification of carbon nanotubes for solubility in organic solvents. Letters to the Editor / Carbon. 2006. 44 (8): 1613.

31. Qingwen L, Jin Z, Hao Y, He M, Liu Z. Thionine-mediated chemistry of carbon nanotubes. Carbon. 2004. 42 (2): 287.

32. Wang AZ, Gu F, Zhang L, Chan JM, Radovic-Moreno A, Shaikh MR, et al. Bio functionalized targeted nanoparticles for therapeutic applications. Expert Opin Biol Ther 2008. 8:1063.

33. Mas-Moruno C, Rechenmacher F. Cilengitide: The first anti-angiogenic small molecule drug candidate, design, synthesis and clinical evaluation. Anticancer Agents Med Chem. 2010. 10: 753.

34. Stupp R1, Hegi ME, Neyns B, Goldbrunner R, Schlegel U, Clement PM, et al. Phase I/IIa study of cilengitide and temozolomide with concomitant radiotherapy followed by cilengitide and temozolomide maintenance therapy in patients with newly diagnosed glioblastoma. J Clin Oncol. 2010. 28: 2712.

35. Dineen SP, Sullivan LA, Beck AW, Miller AF, Carbon JG, Mamluk R, et al. The Adnectin CT-322 is a novel VEGF receptor 2 inhibitor that decreases tumor burden in an orthotopic mouse model of pancreatic cancer. BMC Cancer. 2008. 8 (352): 1.

36. Chu DS, Bocek MJ, Shi J, Ta A, Ngambenjawong C, Rostomily RC, et al. Multivalent display of pendant pro-apoptotic peptides increases cytotoxic activity. J Control Release. 2015. 205: 155.

37. Andre F, Xia W, Conforti R, Wei $Y$, Boulet T, Tomasic G, et al. CXCR4 expression in early breast cancer and risk of distant recurrence. The Oncologist. 2009. 14: 1182.

38. Scala S, Ottaiano A, Ascierto PA, Cavalli M, Simeone E, Giuliano P, et al. Expression of CXCR4 predicts poor prognosis in patients with malignant melanoma. Clin Cancer Res. 2005. 11 (5): 1835.

39. Stevenson CB, Ehtesham M, McMillan KM, Valadez JG, Edgeworth ML, Price RR, et al. CXCR4 expression is elevated in glioblastoma multiforme and correlates with an increase in intensity and extent of peritumoral T2-weighted magnetic resonance imaging signal abnormalities. Neurosurgery. 2008. 63 (3): 560.

40. Hwang JH, Hwang JH, Chung HK, Kim DW, Hwang ES, Suh JM, et al. CXC chemokine receptor 4 expression and function in human anaplastic thyroid cancer cells. J Clin Endocrinol Metab. 2003. 88 (1): 408.

41. Spano JP, Andre F, Morat L, Sabatier L, Besse B, Combadiere C, et al. Chemokine receptor CXCR4 and early-stage non-small cell lung cancer: 
pattern of expression and correlation with outcome. Ann Oncol. 2004. 15: 613.

42. Wehler T, Wolfert F, Schimanski CC, Gockel I, Herr W, Biesterfeld S, et al. Strong expression of chemokine receptor CXCR4 by pancreatic cancer correlates with advanced disease. Oncol Rep. 2006. 16 (611): 59.

43. Jiang $\mathrm{YP}, \mathrm{Wu} \mathrm{XH}, \mathrm{Shi} \mathrm{B}, \mathrm{Wu} \mathrm{WX}$, Yin GR. Expression of chemokine CXCL12 and its receptor CXCR4 in human epithelial ovarian cancer: an independent prognostic factor for tumor progression. Gynecol Oncol. 2006. 103 (1): 226.

44. Akashi T, Koizumi K, Tsuneyama K, Saiki I, Takano Y, Fuse H. Chemokine receptor CXCR4 expression and prognosis in patients with metastatic prostate cancer. Cancer Sci. 2008. 99 (3): 539.

45. Kim J, Takeuchi H, Lam ST, Turner RR, Wang HJ, Kuo C, et al. Chemokine receptor CXCR4 expression in colorectal cancer patients increases the risk for recurrence and for poor survival. J Clin Oncol. 2005. 23: 2744 . 